\title{
Total Synthesis of Heliophenanthrone
}

\author{
Gerald Dyker* and Dirk Hildebrandt
}

Fakultät für Chemie, Ruhr-Universität Bochum

Universitätsstraße 150, D-44780 Bochum, Germany

\section{List of Contents:}

Preparation of 2-[3-(2-Formyl-phenyl)-prop-2-ynyl]-2-prop-2-ynyl-malonic acid diethyl ester $\mathbf{1}$ 


\section{Experimental Section}

General. Unless otherwise noted, all reactions were carried out under argon atmosphere in oven-dried glassware. All solvents were dried and distilled. Flash-column chromatography was performed on silica gel. Melting points are uncorrected. NMR spectra were recorded in $\mathrm{CDCl}_{3}$, at $400 \mathrm{MHz}\left({ }^{1} \mathrm{H}\right)$ and 100 $\mathrm{MHz}\left({ }^{13} \mathrm{C}\right) ; \delta$ in $\mathrm{ppm}$ relative to solvent peak or TMS. Infrared spectra (IR) are reported in wavenumbers $\left(\mathrm{cm}^{-1}\right)$. Mass spectra were obtained using electron ionisation (EI). HRMS spectra were obtained using electrospray ionisation (ESI).

Preparation of 2-[3-(2-Formyl-phenyl)-prop-2-ynyl]-malonic acid diethyl ester S3:<smiles>C#CCC(C(=O)OCC)C(=O)OCC</smiles>

S1<smiles>O=Cc1ccccc1Br</smiles>

S2

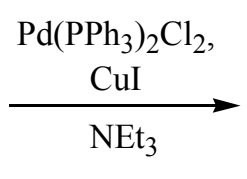

S3

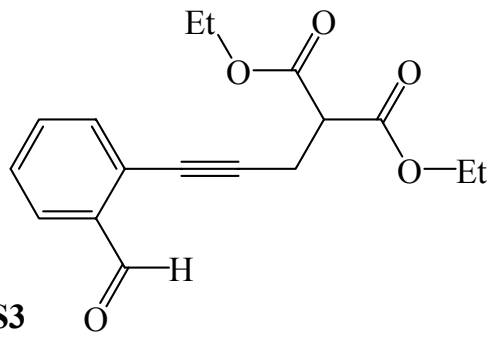

In a screw capped flask $648 \mathrm{mg}(3.50 \mathrm{mmol})$ of 2-bromobenzaldehyd (S2), $594 \mathrm{mg}(3.00 \mathrm{mmol})$ of propargyl-malonic acid ester S1, $105 \mathrm{mg}(0.15 \mathrm{mmol}, 5 \mathrm{~mol} \%)$ of $\mathrm{PdCl}_{2}\left(\mathrm{PPh}_{3}\right)_{2}$ and $14.0 \mathrm{mg}(0.08$ mmol, $2.5 \mathrm{~mol} \%$ ) of $\mathrm{CuI}$ were suspended in $20 \mathrm{ml}$ of triethylamine. The mixture was stirred and heated at $80^{\circ} \mathrm{C}$ for $8 \mathrm{~h}$. The suspension was filtered through a short silica pad with ethyl acetate. Evaporation of the solvents and flash chromatography of the residue $\left(\mathrm{SiO}_{2}\right.$, petroleum ether/MTBE $\left.(4: 1) \mathrm{R}_{f}=0.27\right)$ gave product $\mathbf{S 3}$ (444 mg, $1.47 \mathrm{mmol}, 49 \%)$ as yellow oil: IR (NaCl): $\widetilde{v}=2986 \mathrm{~cm}^{-1}(\mathrm{~m}), 2941(\mathrm{~m})$, 2238 (w), 1736 (vs), 1697 (vs), 1595 (s), 1271 (s), 1176 (s), 1034 (s), 766 (s); ${ }^{1} \mathrm{H}$ NMR (CDCl 3,400 MHz): $\delta=1.29 \mathrm{ppm}(\mathrm{t}, J=7.0 \mathrm{~Hz}, 6 \mathrm{H}), 3.08(\mathrm{~d}, J=8.0 \mathrm{~Hz}, 2 \mathrm{H}), 3.68(\mathrm{t}, J=7.5 \mathrm{~Hz}, 1 \mathrm{H}), 4.25(\mathrm{q}, J=$ $7.0 \mathrm{~Hz}, 4 \mathrm{H}), 7.40(\mathrm{t}, J=7.0 \mathrm{~Hz}, 1 \mathrm{H}), 7.47-7.53(\mathrm{~m}, 2 \mathrm{H}), 7.88(\mathrm{~d}, J=8.0 \mathrm{~Hz}, 1 \mathrm{H}), 10.46(\mathrm{~s}, 1 \mathrm{H}) ;{ }^{13} \mathrm{C}$ $\operatorname{NMR}\left\{{ }^{1} \mathrm{H}\right\}\left(\mathrm{CDCl}_{3}, 100 \mathrm{MHz}\right): \delta=14.19 \mathrm{ppm}, 19.75,51.29,62.07$, 78.37, 93.20, 127.15, 128.56, 133.49, 133.79, 136.36, 168.01, 191.92; MS (70 eV, EI): $m / z(\%)=302\left[\mathrm{M}^{+}\right](6), 273(100), 227(35)$, 210 (19), 199 (25), 183 (29), 145 (22), 127 (31), 115 (33), 77 (12); Anal. Calcd $\mathrm{C}_{17} \mathrm{H}_{18} \mathrm{O}_{5}$ : C, 67.54; H, 6.00. Found: C, 67.15; H, 5.84. 
Preparation of 2-[3-(2-Formyl-phenyl)-prop-2-ynyl]-2-prop-2-ynyl-malonic acid diethyl ester 1:
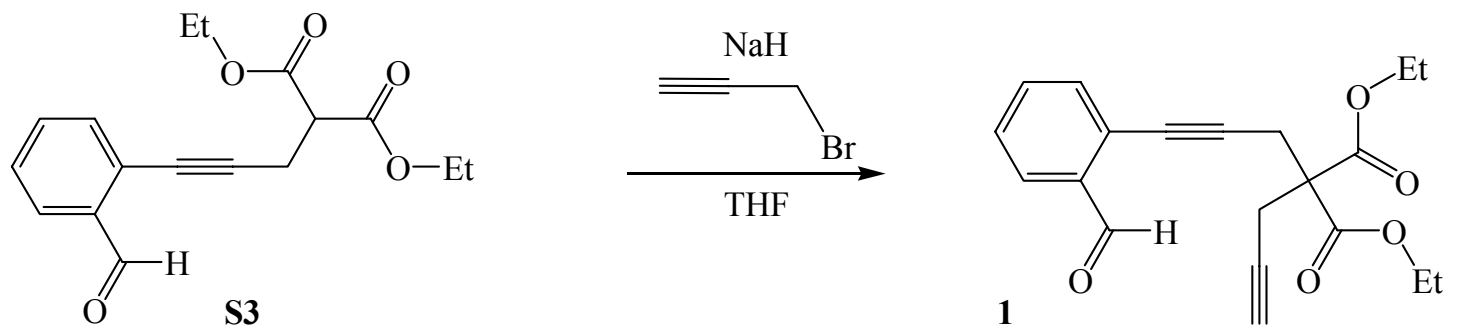

To a ice cooled suspension of $38.0 \mathrm{mg}(1.60 \mathrm{mmol}) \mathrm{NaH}$ in $5 \mathrm{ml}$ of THF were added dropwise $302 \mathrm{mg}$ $(1.00 \mathrm{mmol})$ of malonic acid ester $\mathbf{S 3}$ in $5 \mathrm{ml}$ of THF. The mixture was stirred for $1 \mathrm{~h}$ at $0^{\circ} \mathrm{C}$. A solution of $179 \mathrm{mg}(1.20 \mathrm{mmol})$ propargyl bromide $(80 \%$ in toluen) in $5 \mathrm{ml}$ of THF were added. Then the mixture was warmed to roomtemperature and stirred over night. $10 \mathrm{ml}$ of water were added and the organic layer was seperated. The aqueous layer was extracted with $10 \mathrm{ml}(3 \mathrm{x})$ of MTBE. The organic extract was washed with brine and dried over sodium sulfate. Evaporation of the solvents and flash chromatography of the residue $\left(\mathrm{SiO}_{2}\right.$, petroleum ether/MTBE (4:1) $\left.\mathrm{R}_{f}=0.34\right)$ gave product 1 (190 mg, $0.56 \mathrm{mmol}, 56 \%$ ) as yellow oil: IR (NaCl): $\widetilde{v}=3290 \mathrm{~cm}^{-1}(\mathrm{~s}), 3070,(\mathrm{~s}), 2987(\mathrm{~m}), 2940(\mathrm{~m}), 2239(\mathrm{w})$, 2124 (w), 1738 (br, vs), 1699 (vs), 1596 (s), 1298 (s), 1249 (s), 1194 (s), 765 (m); ${ }^{1} \mathrm{H} \mathrm{NMR} \mathrm{(CDCl}{ }_{3}, 400$ MHz): $\delta=1.28 \mathrm{ppm}(\mathrm{t}, J=7.0 \mathrm{~Hz}, 6 \mathrm{H}), 2.07(\mathrm{t}, J=2.5 \mathrm{~Hz}, 1 \mathrm{H}), 3.04(\mathrm{~d}, J=2.5 \mathrm{~Hz}, 2 \mathrm{H}), 3.30(\mathrm{~s}, 2 \mathrm{H})$, $4.26(\mathrm{q}, J=7.0 \mathrm{~Hz}, 4 \mathrm{H}), 7.41(\mathrm{td}, J=7.0,2.0 \mathrm{~Hz}, 1 \mathrm{H}), 7.48-7.54(\mathrm{~m}, 2 \mathrm{H}), 7.88(\mathrm{~d}, J=7.5 \mathrm{~Hz}, 1 \mathrm{H})$, $10.45(\mathrm{~d}, J=1.0 \mathrm{~Hz}, 1 \mathrm{H}) ;{ }^{13} \mathrm{C} \mathrm{NMR}\left\{{ }^{1} \mathrm{H}\right\}\left(\mathrm{CDCl}_{3}, 100 \mathrm{MHz}\right): \delta=14.18 \mathrm{ppm}, 23.11,23.93,56.64$, 62.34, 72.08, 78.49, 79.57, 91.65, 126.77, 127.24, 128.63, 133.73, 133.79, 136.36, 168.78, 191.82; MS (70 eV, EI): $m / z(\%)=340\left[\mathrm{M}^{+}\right](9), 311$ (47), 267 (24), 239 (18), 221 (12), 193 (77), 165 (62), 145 (24), 115 (42), 89 (14), 77 (10), 29 (100); HRMS (ESI) calcd for $\mathrm{C}_{20} \mathrm{H}_{21} \mathrm{O}_{5}\left(\mathrm{M}^{+}+\mathrm{H}\right)$ 341.1384, found 341.1379 . 
NMR-Spectra:

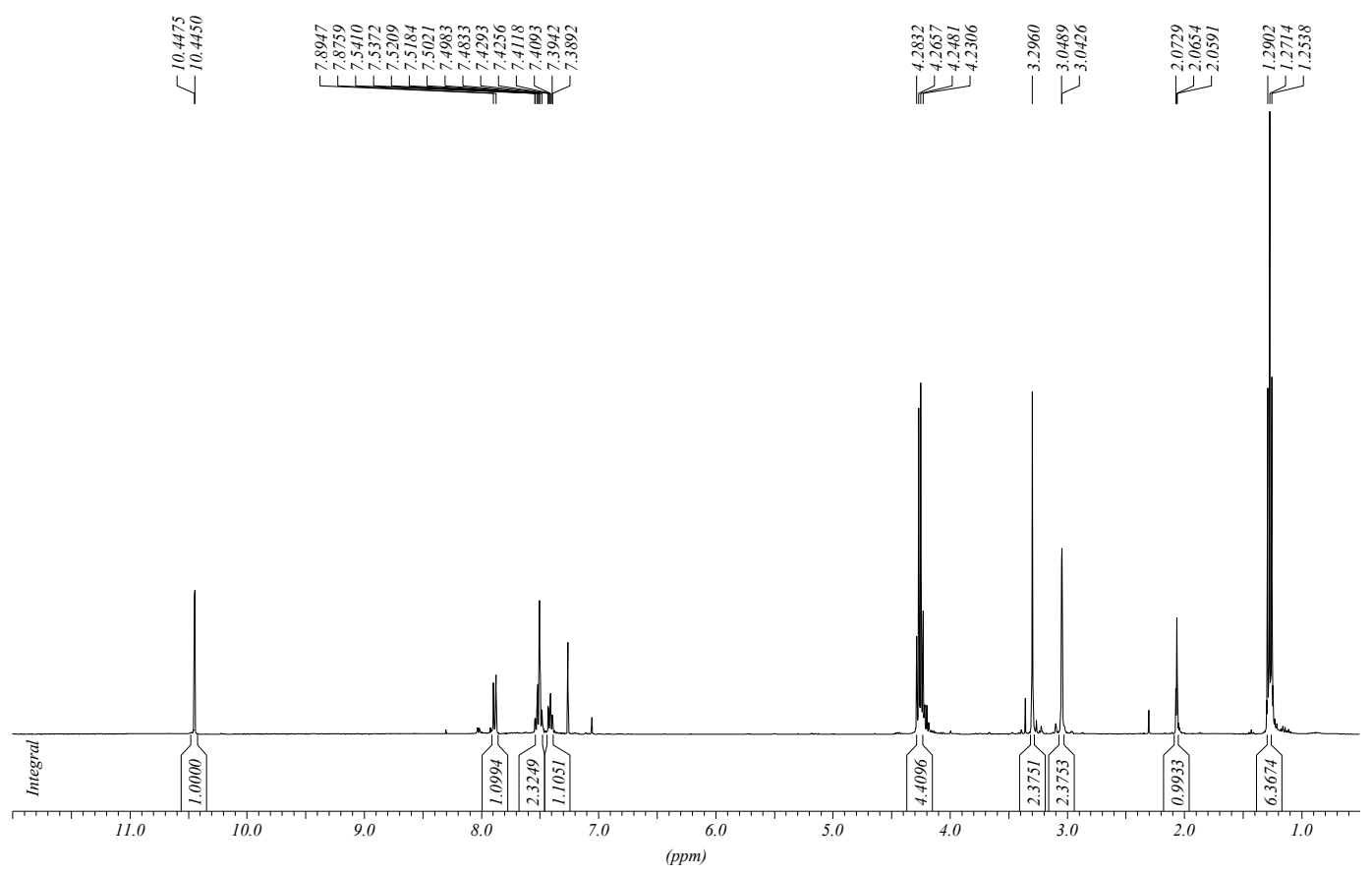

${ }^{1} \mathrm{H}$ NMR (400MHz, $\mathrm{CDCl}_{3}$ ): 2-[3-(2-Formyl-phenyl)-prop-2-ynyl]-malonic acid diethyl ester (S3)

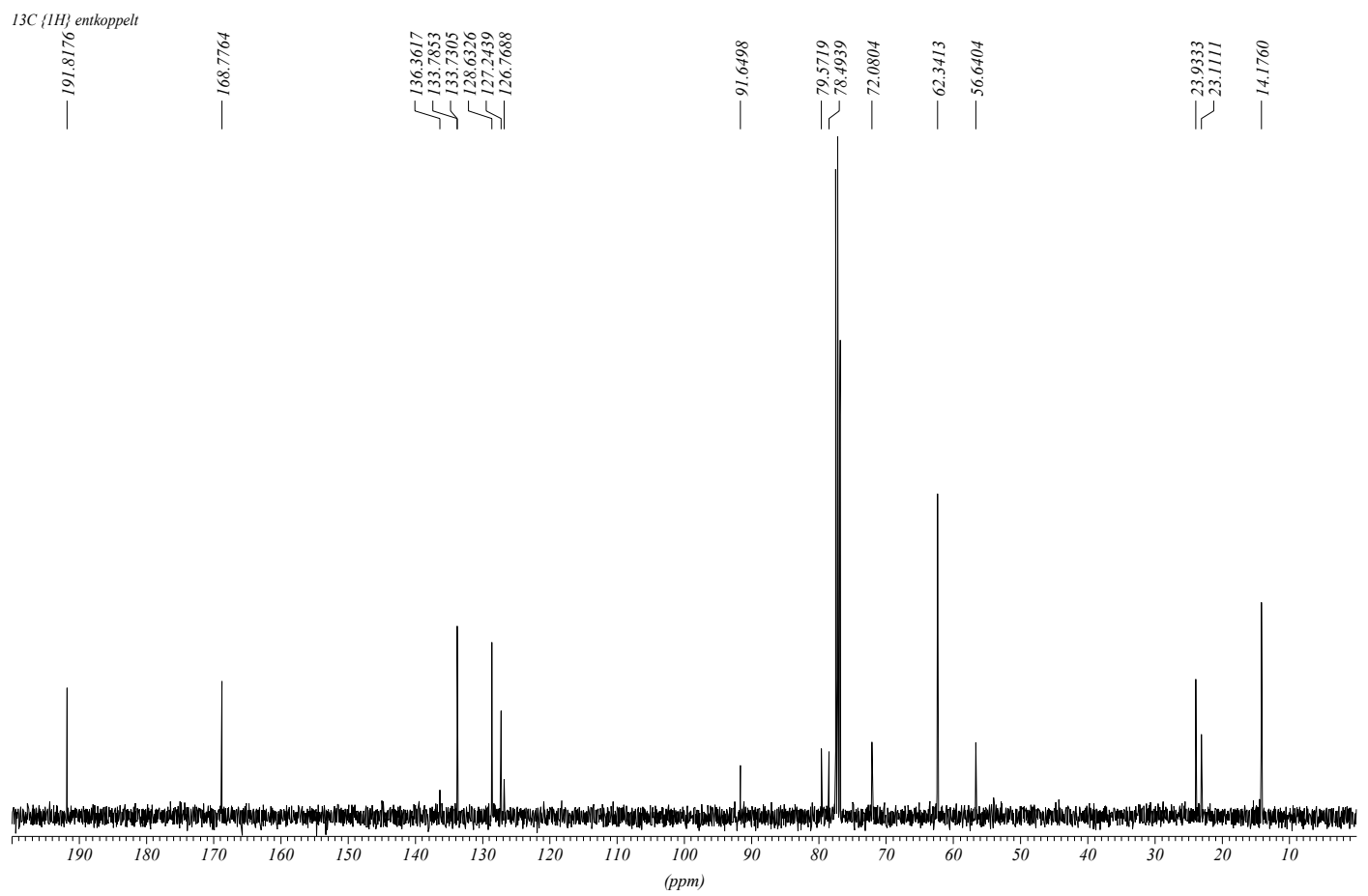

${ }^{13} \mathrm{C}$ NMR (100MHz, $\mathrm{CDCl}_{3}$ ): 2-[3-(2-Formyl-phenyl)-prop-2-ynyl]-malonic acid diethyl ester (S3) 


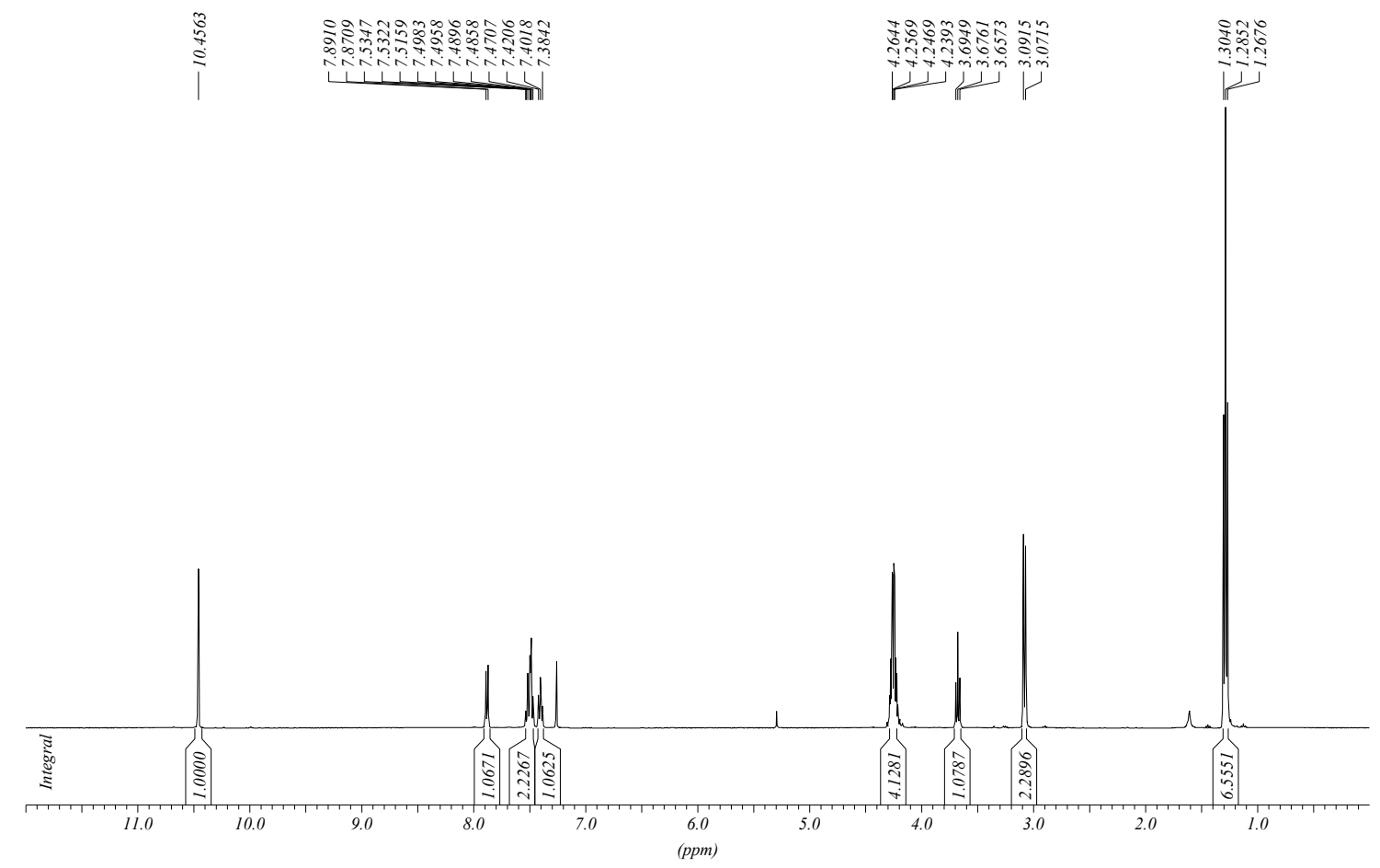

${ }^{1} \mathrm{H}$ NMR (400MHz, $\mathrm{CDCl}_{3}$ ): 2-[3-(2-Formyl-phenyl)-prop-2-ynyl]-2-prop-2-ynyl-malonic acid diethyl ester (1)

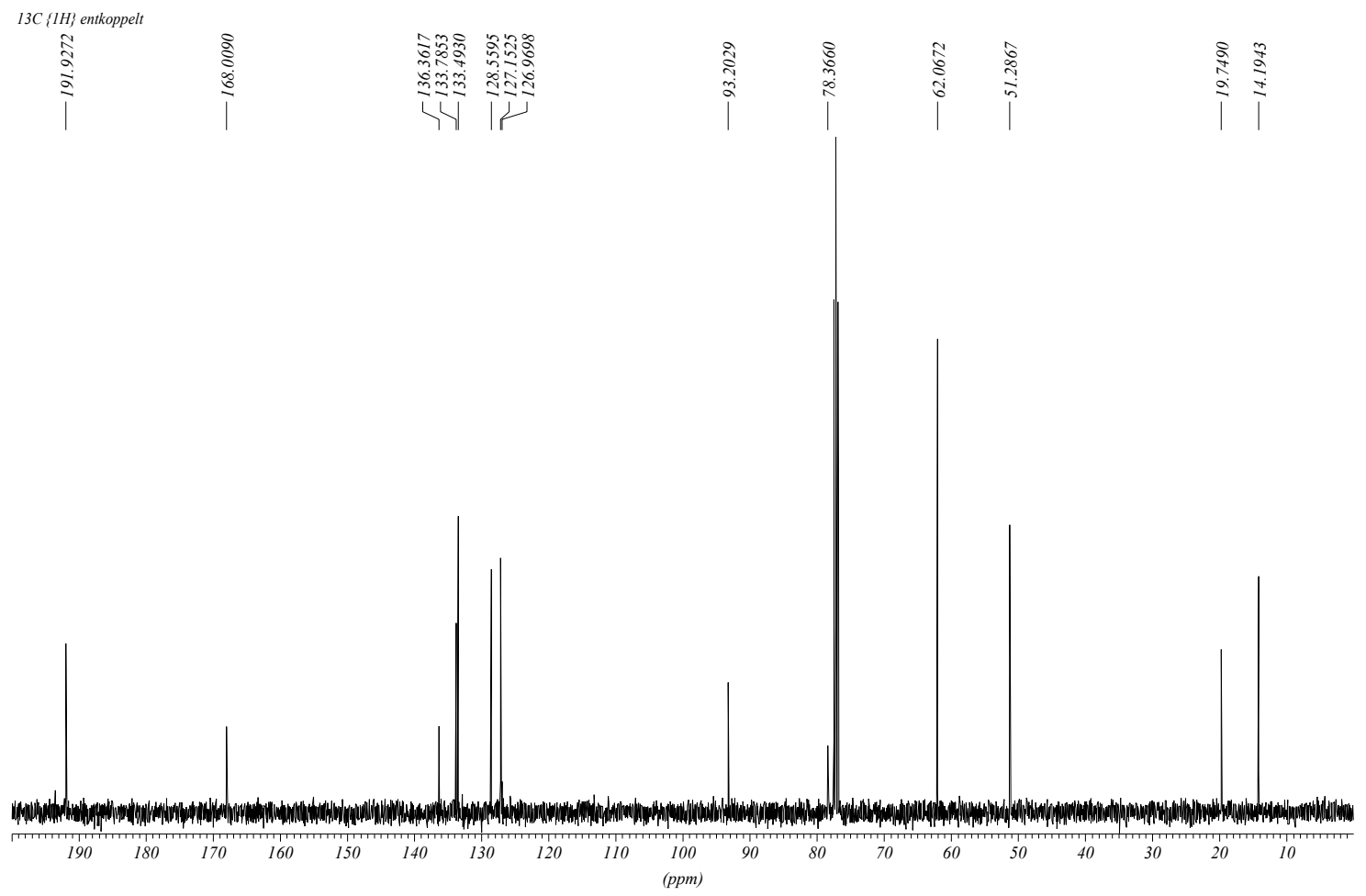

${ }^{13} \mathrm{C}$ NMR (100MHz, $\mathrm{CDCl}_{3}$ ): 2-[3-(2-Formyl-phenyl)-prop-2-ynyl]-2-prop-2-ynyl-malonic acid diethyl ester (1) 


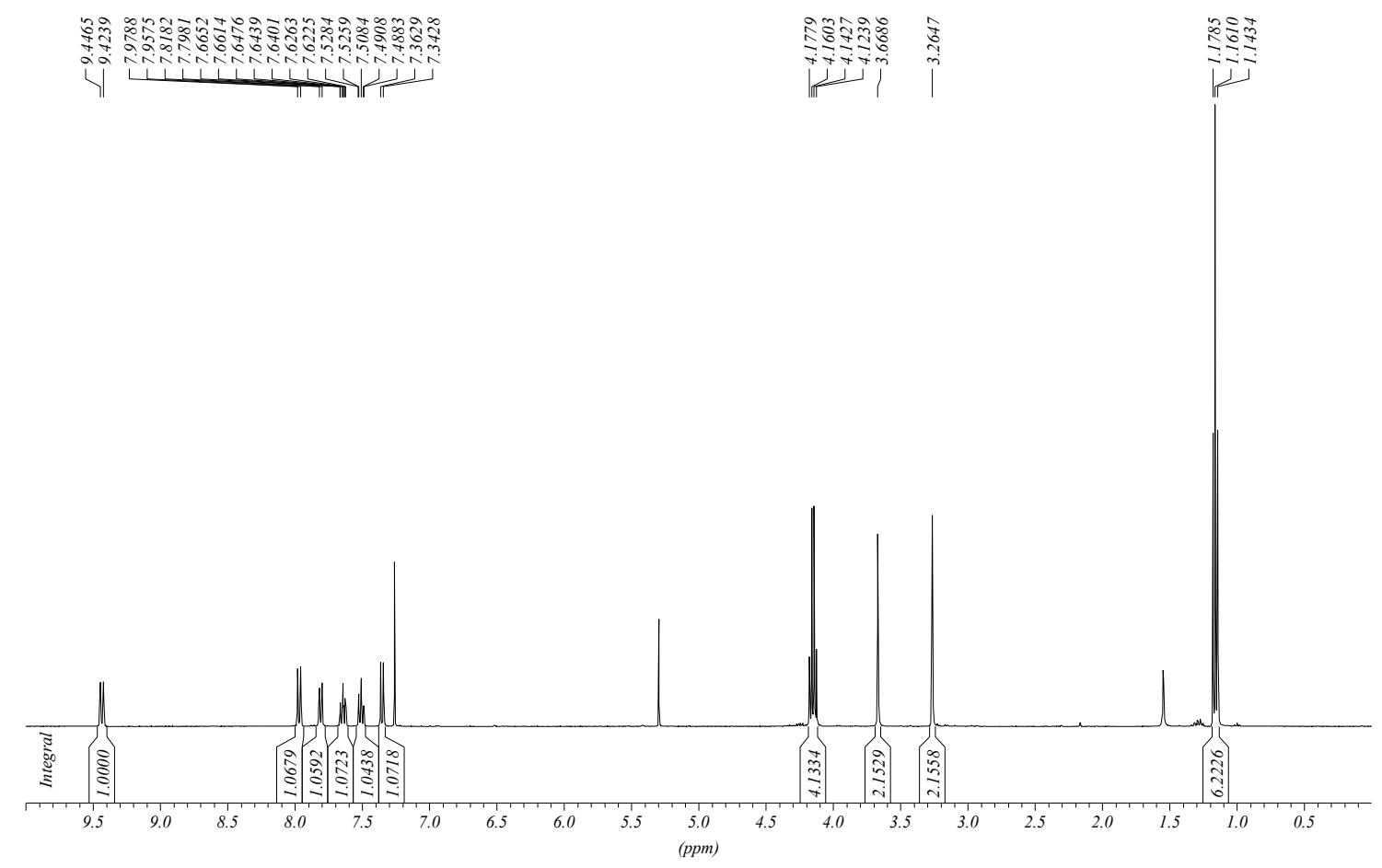

${ }^{1} \mathrm{H}$ NMR (400MHz, $\mathrm{CDCl}_{3}$ ): 4-Oxo-3,4-dihydro-1H-phenanthrene-2,2-dicarboxylic acid diethyl ester (2)

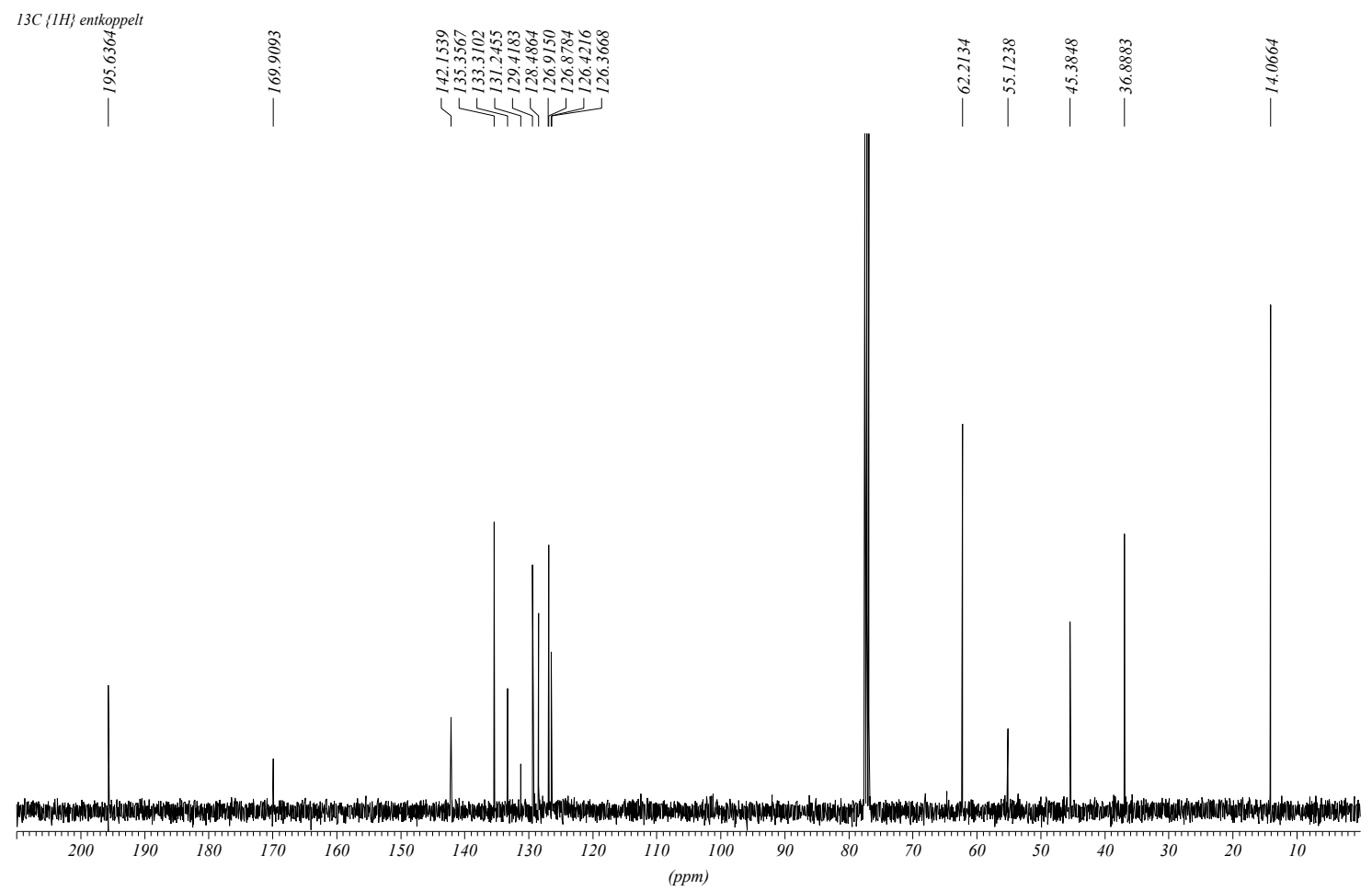

${ }^{13} \mathrm{C}$ NMR (100MHz, $\mathrm{CDCl}_{3}$ ): 4-Oxo-3,4-dihydro-1H-phenanthrene-2,2-dicarboxylic acid diethyl ester (2) 


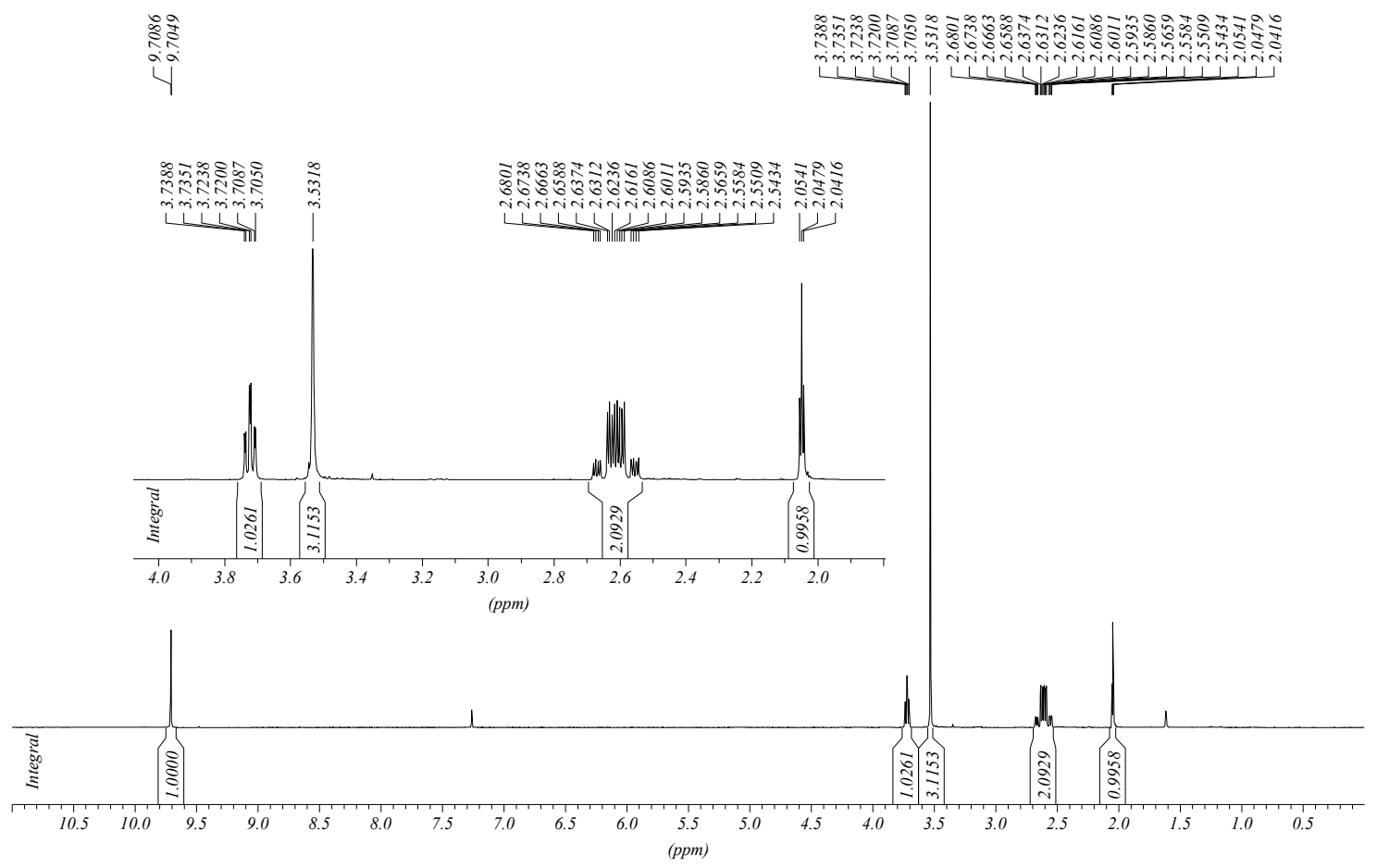

${ }^{1} \mathrm{H}$ NMR (400MHz, $\mathrm{CDCl}_{3}$ ): 2-Methoxypent-4-ynal (10)

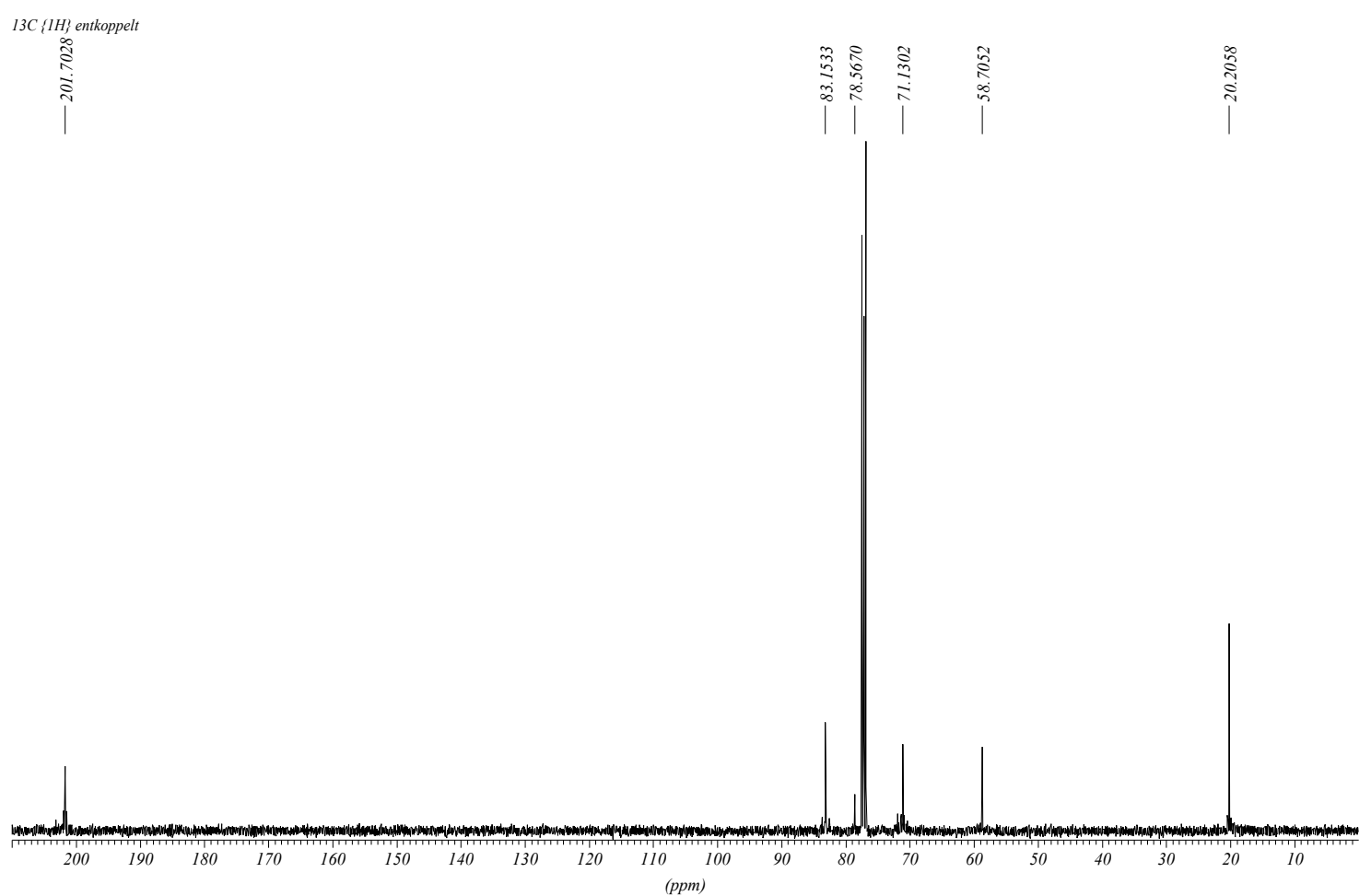

${ }^{13} \mathrm{C}$ NMR (100MHz, $\mathrm{CDCl}_{3}$ ): 2-Methoxypent-4-ynal (10) 


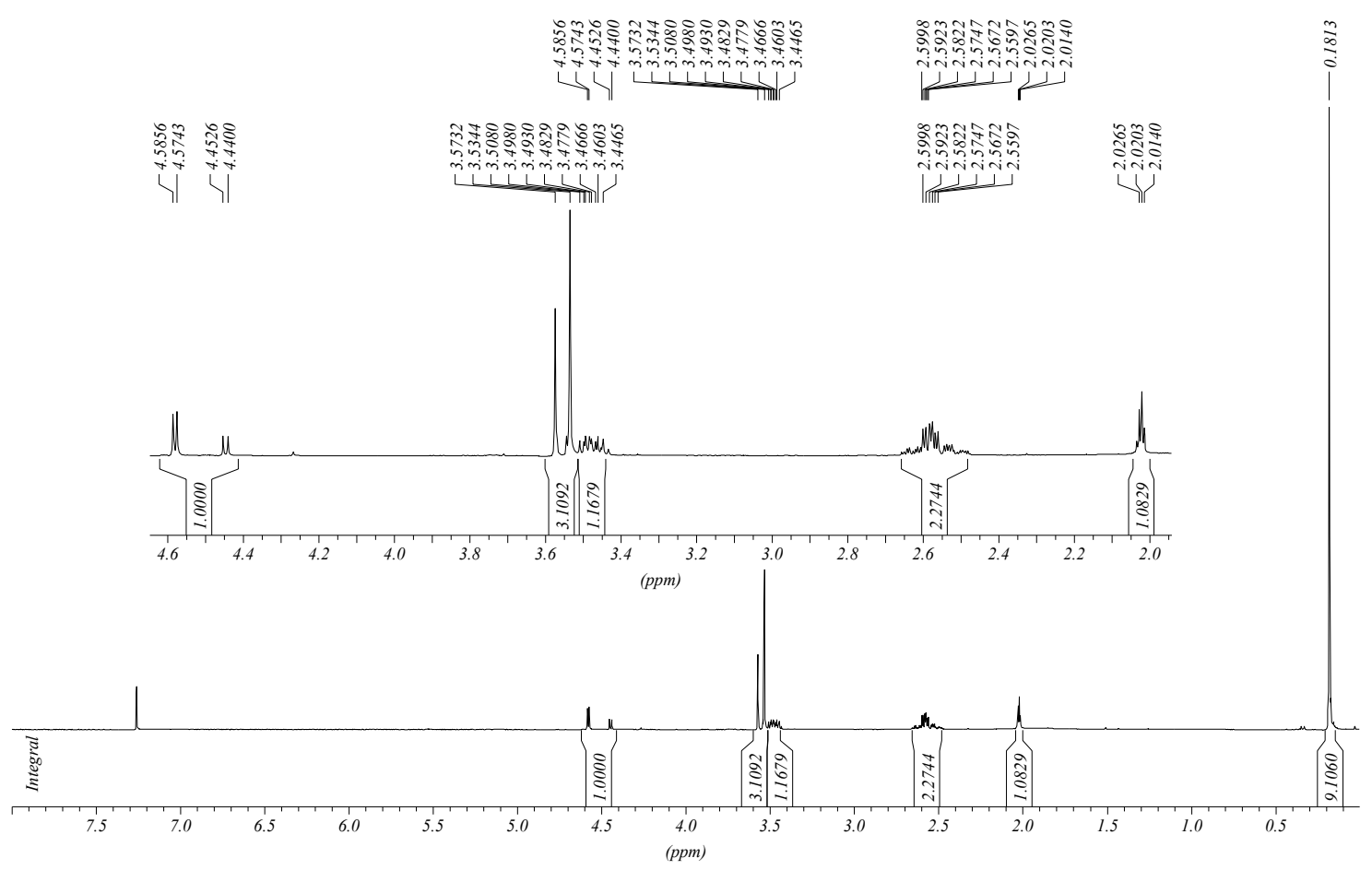

${ }^{1} \mathrm{H}$ NMR (400MHz, $\mathrm{CDCl}_{3}$ ): 4-Methoxy-1-trimethylsilanyl-hepta-1,6-diyn-3-ol (6a/b)

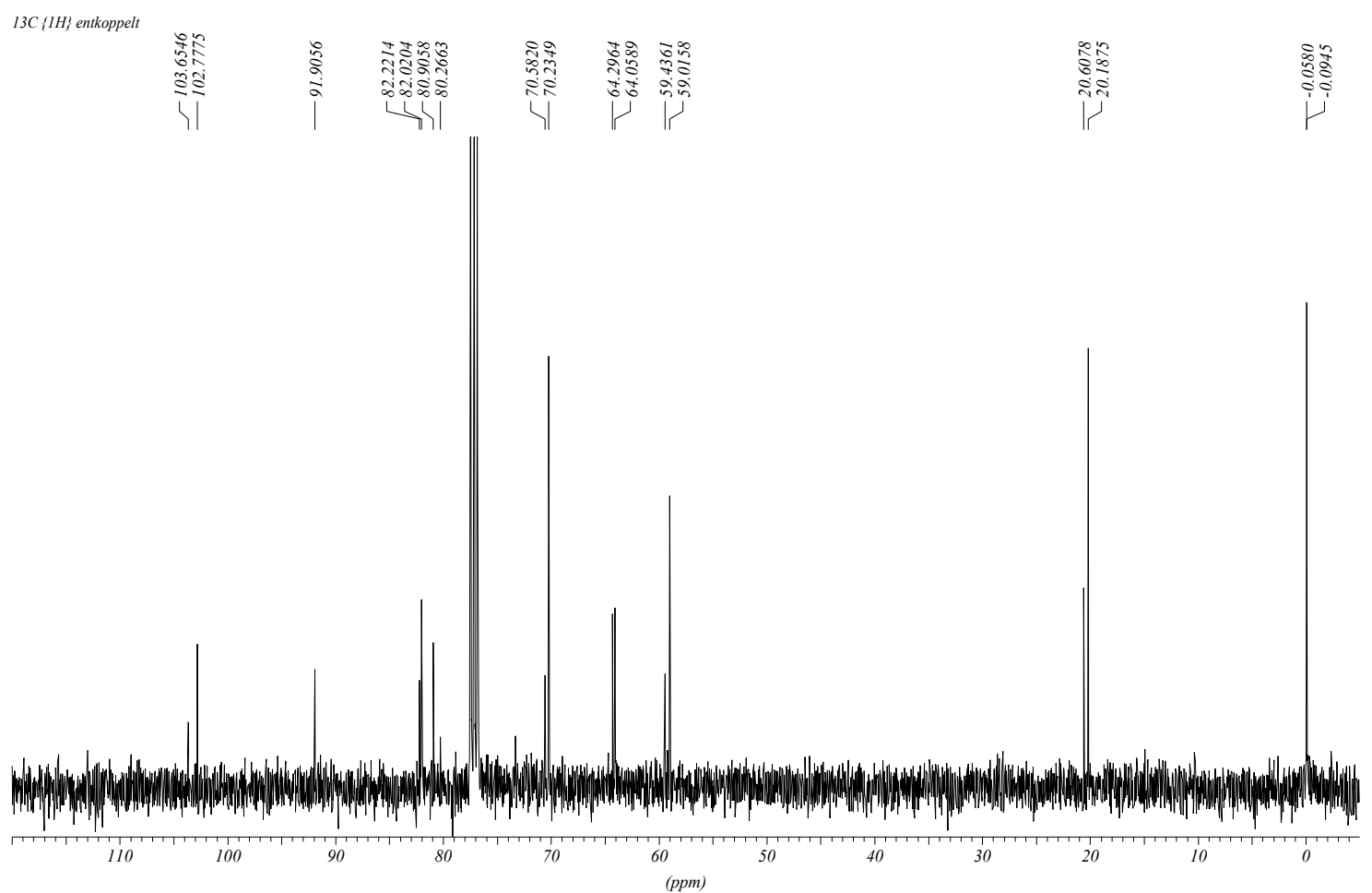

${ }^{13} \mathrm{C}$ NMR (100MHz, $\mathrm{CDCl}_{3}$ ): 4-Methoxy-1-trimethylsilanyl-hepta-1,6-diyn-3-ol (6a/b) 


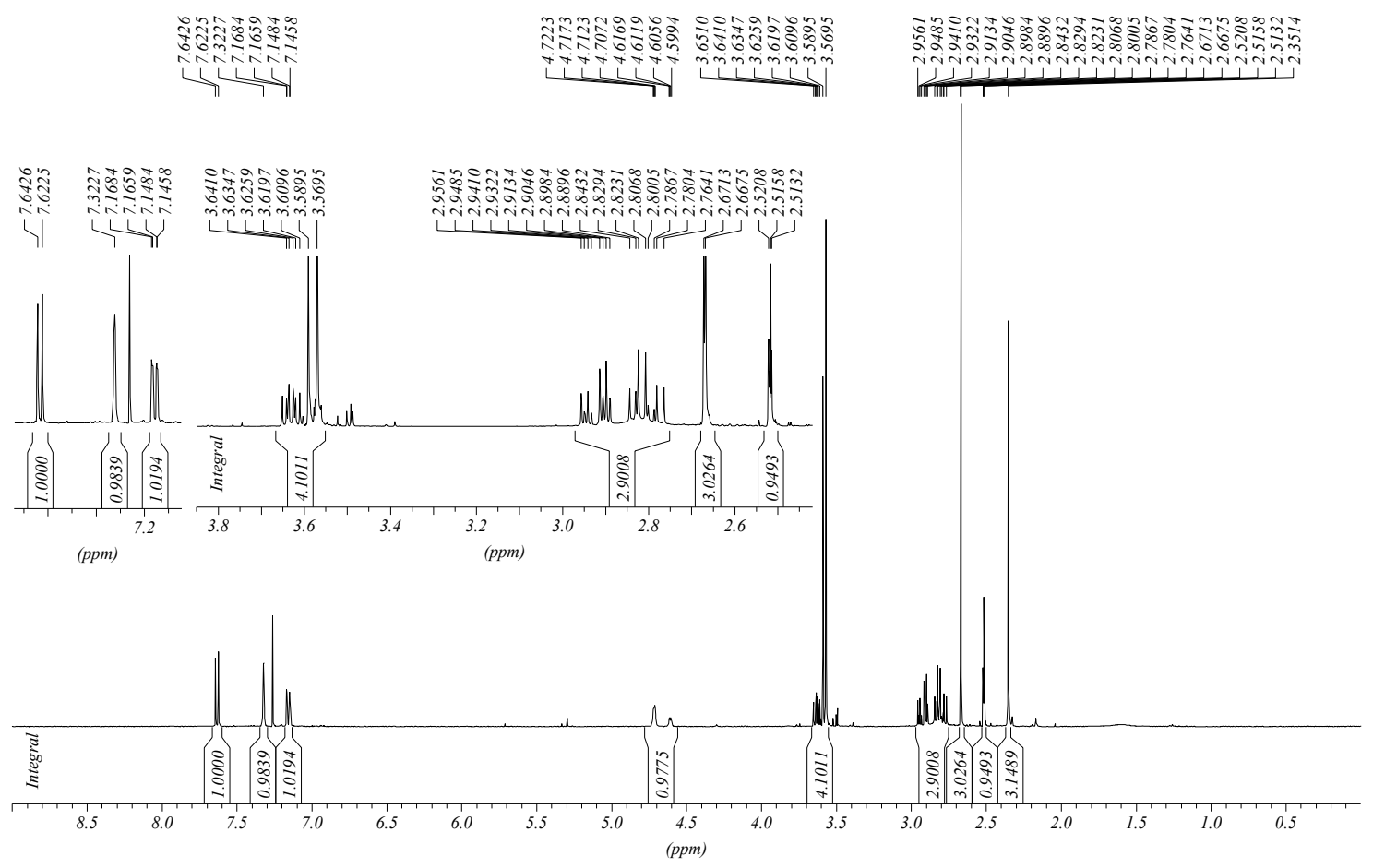

${ }^{1} \mathrm{H}$ NMR (400MHz, CDCl 3 ): 1-[2-(5-Hydroxy-4-methoxy-hepta-1,6-diynyl)-4-methyl-phenyl]-ethanone (4a/b)

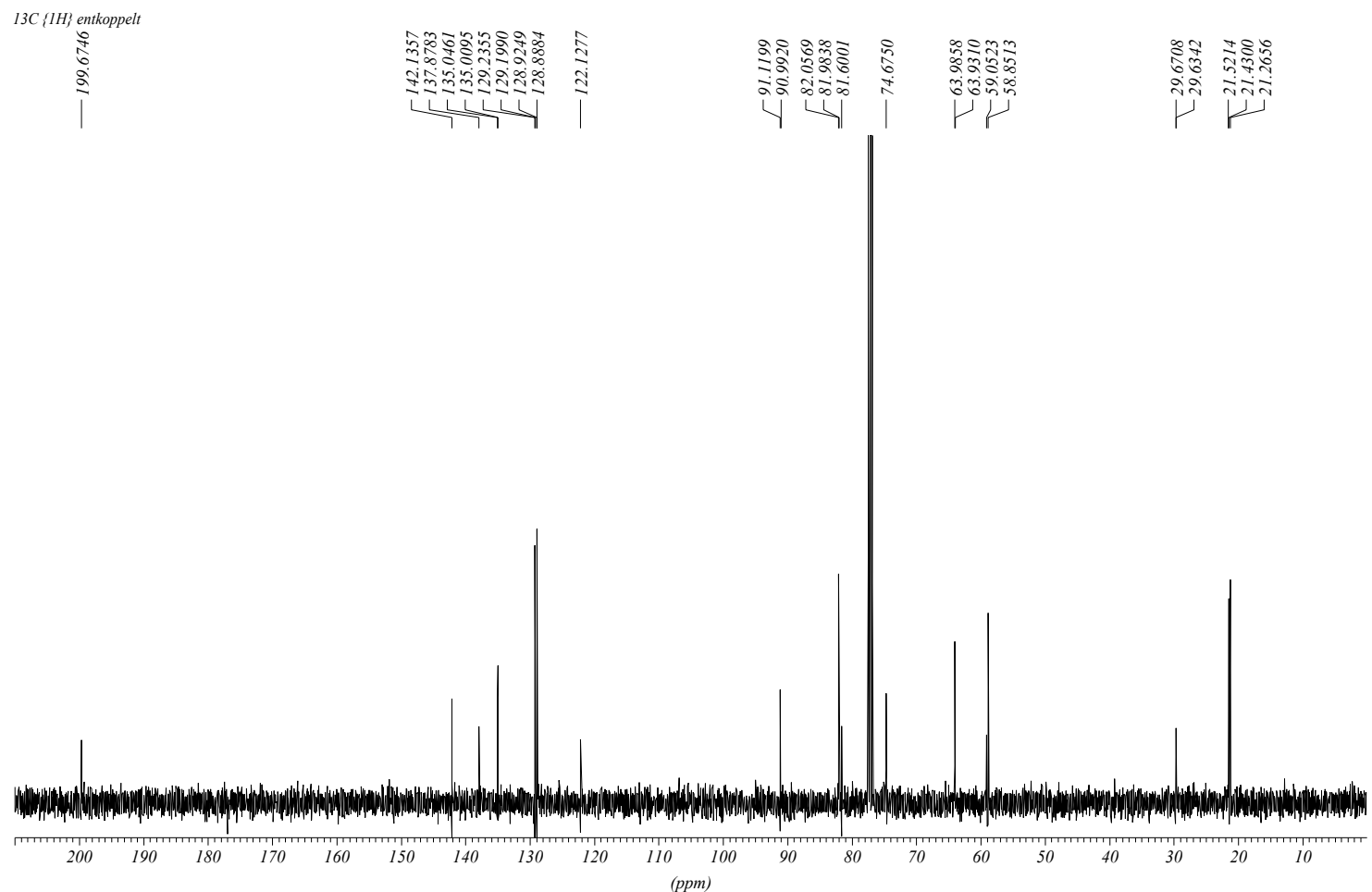

${ }^{13} \mathrm{C}$ NMR (100MHz, $\left.\mathrm{CDCl}_{3}\right):$ 1-[2-(5-Hydroxy-4-methoxy-hepta-1,6-diynyl)-4-methyl-phenyl]-ethanone (4a/b) 


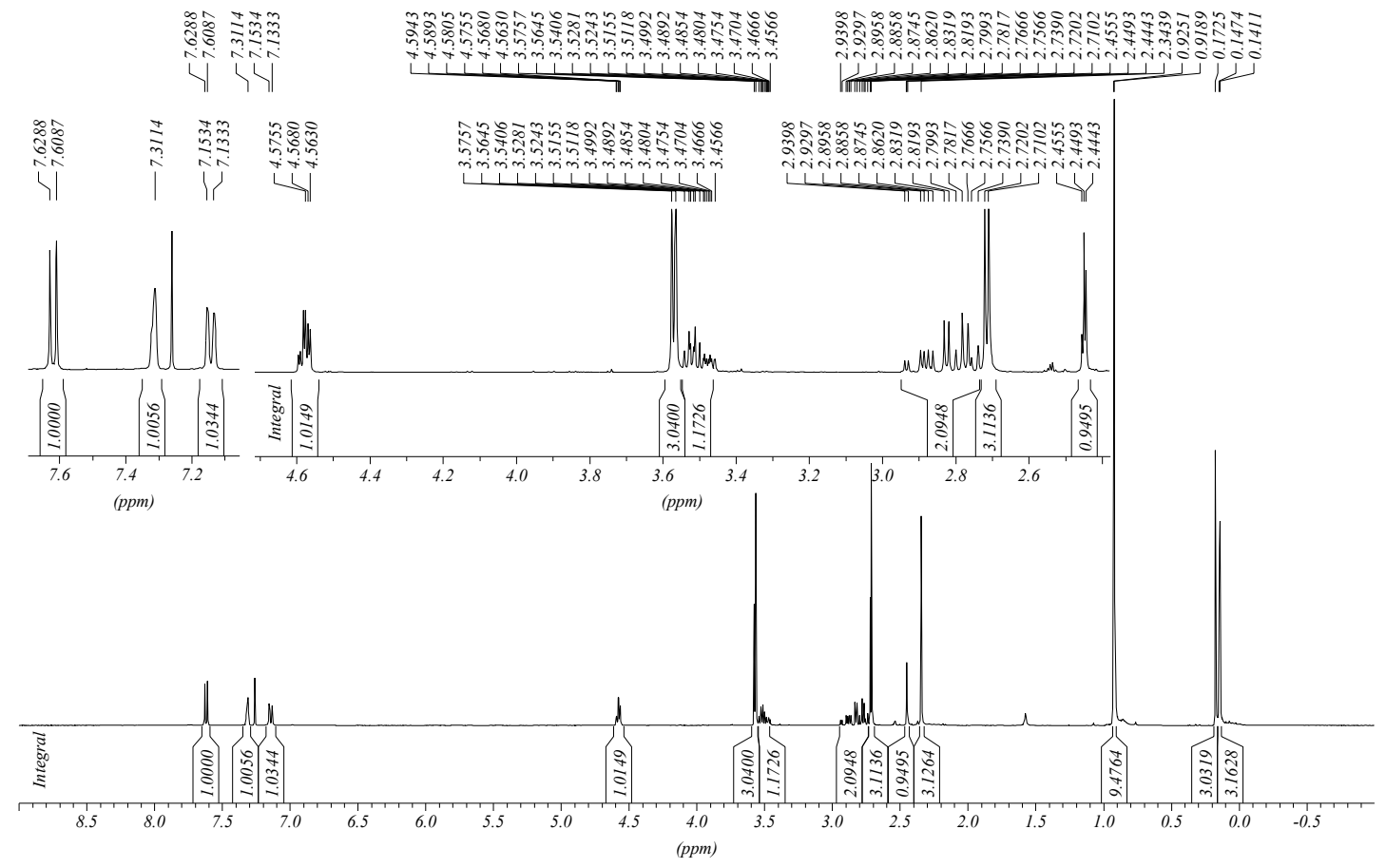

${ }^{1} \mathrm{H} \quad \mathrm{NMR} \quad\left(400 \mathrm{MHz}, \quad \mathrm{CDCl}_{3}\right)$ : 1-\{2-[5-(tert-Butyl-dimethyl-silanyloxy)-4-methoxy-hepta-1,6-diynyl]-4-methyl-phenyl $\}$ ethanone $(\mathbf{1 2 a} / \mathbf{b})$

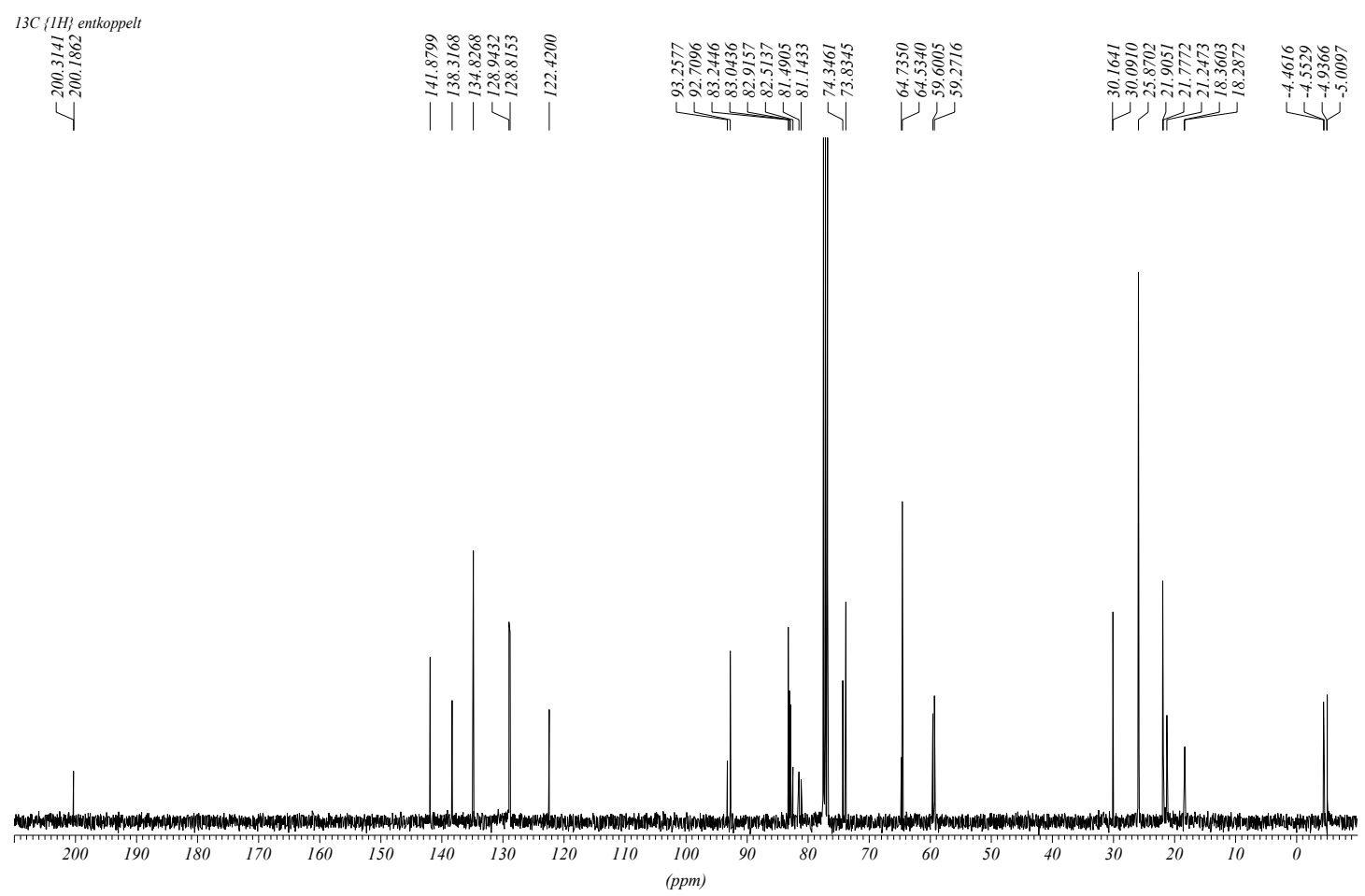

${ }^{13} \mathrm{C}$ NMR $\left(100 \mathrm{MHz}, \quad \mathrm{CDCl}_{3}\right):$ 1-\{2-[5-(tert-Butyl-dimethyl-silanyloxy)-4-methoxy-hepta-1,6-diynyl]-4-methyl-phenyl\}ethanone $(\mathbf{1 2 a} / \mathbf{b})$ 


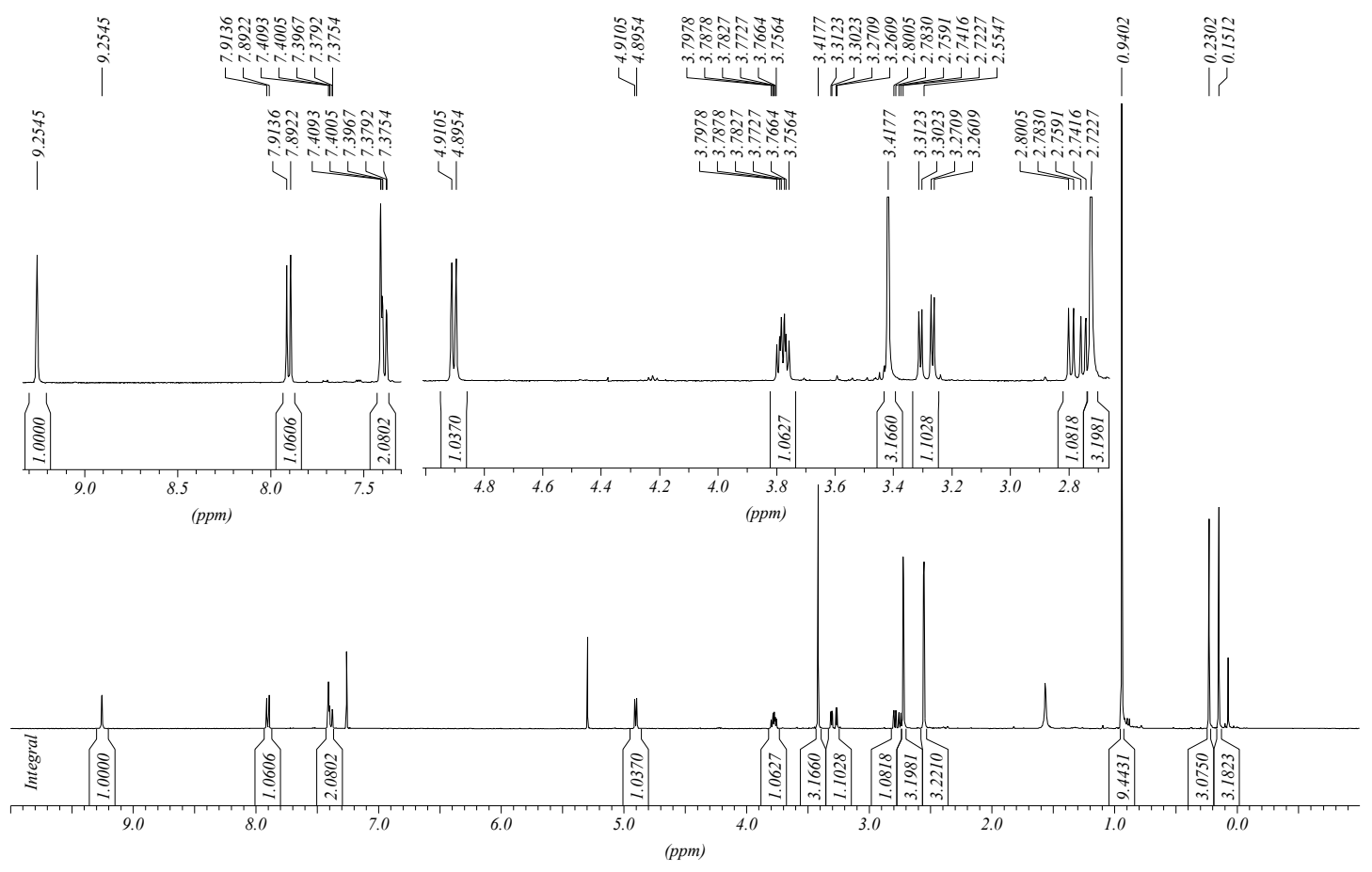

${ }^{1} \mathrm{H}$ NMR (400MHz, $\mathrm{CDCl}_{3}$ ): 1-(tert-Butyl-dimethyl-silanyloxy)-2-methoxy-6,9-dimethyl-2,3-dihydro-1H-phenanthren-4one (11a) 


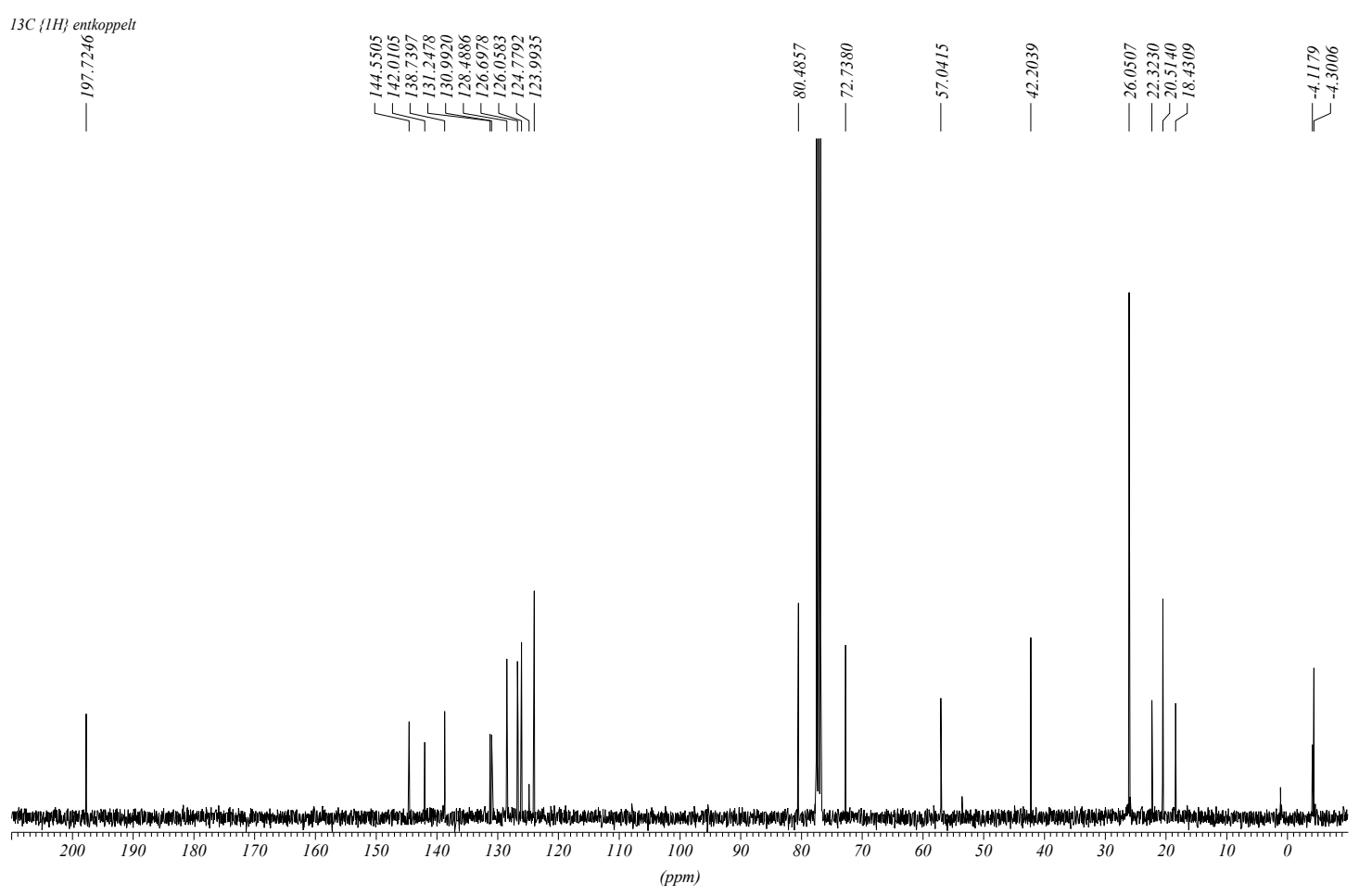

${ }^{13} \mathrm{C}$ NMR (100MHz, $\left.\mathrm{CDCl}_{3}\right)$ : 1-(tert-Butyl-dimethyl-silanyloxy)-2-methoxy-6,9-dimethyl-2,3-dihydro-1H-phenanthren-4one (11a)

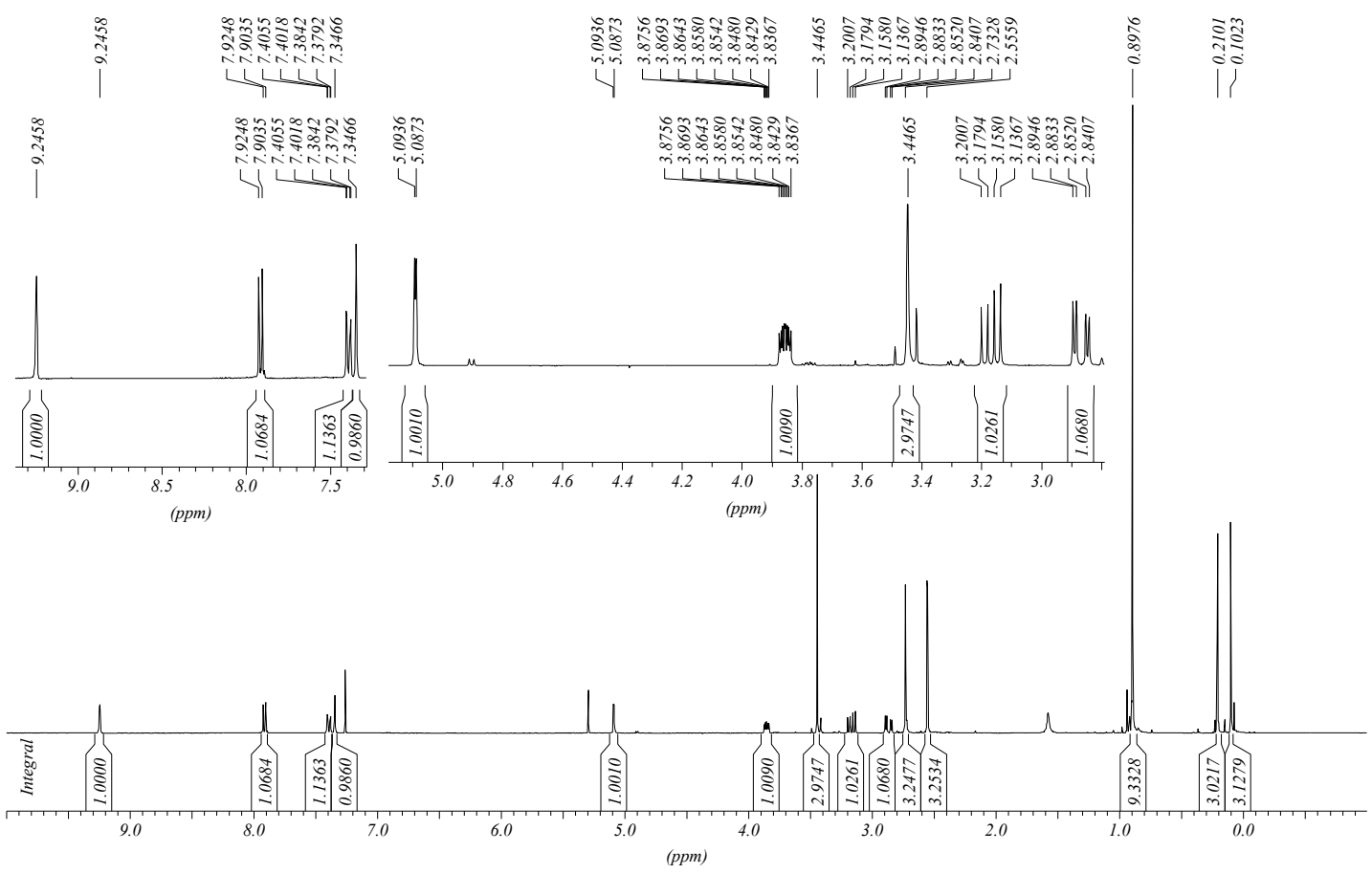

${ }^{1} \mathrm{H}$ NMR (400MHz, $\mathrm{CDCl}_{3}$ ): 1-(tert-Butyl-dimethyl-silanyloxy)-2-methoxy-6,9-dimethyl-2,3-dihydro-1H-phenanthren-4one (11b) 


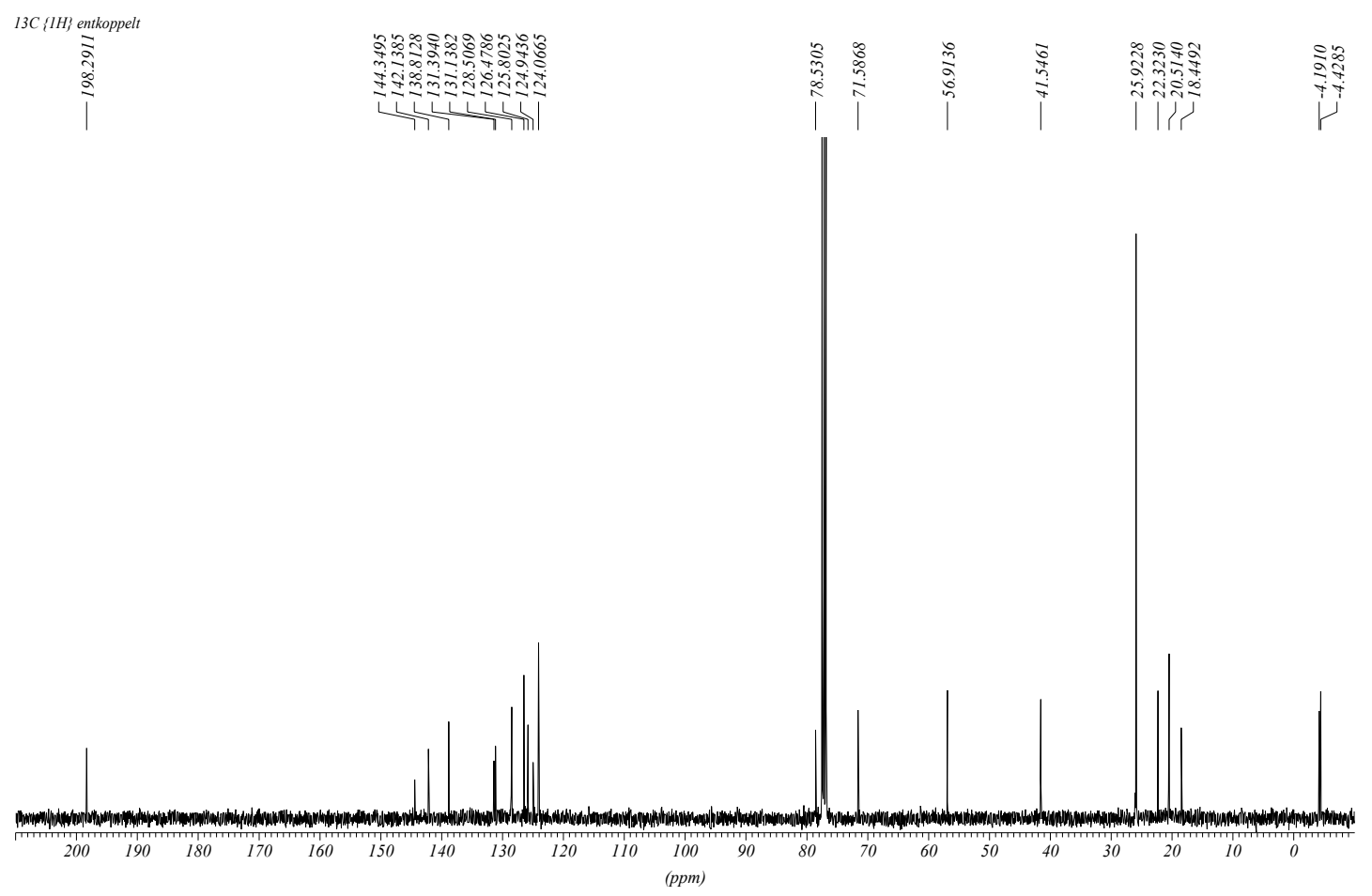

${ }^{13} \mathrm{C}$ NMR (100MHz, $\left.\mathrm{CDCl}_{3}\right)$ : 1-(tert-Butyl-dimethyl-silanyloxy)-2-methoxy-6,9-dimethyl-2,3-dihydro-1H-phenanthren-4one (11b)

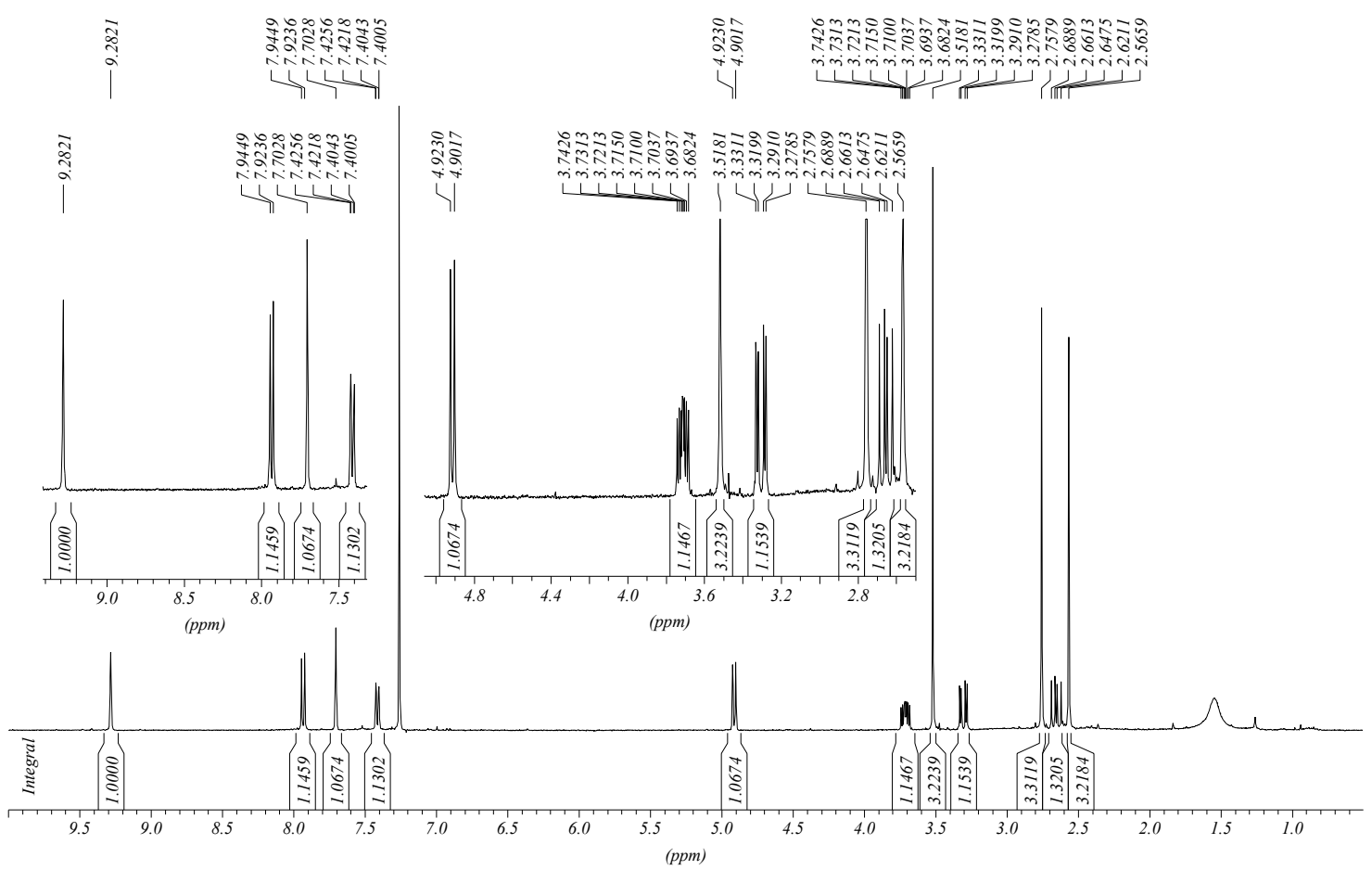

${ }^{1} \mathrm{H}$ NMR (400MHz, $\left.\mathrm{CDCl}_{3}\right): r a c$-Heliophenanthrone (3a) 


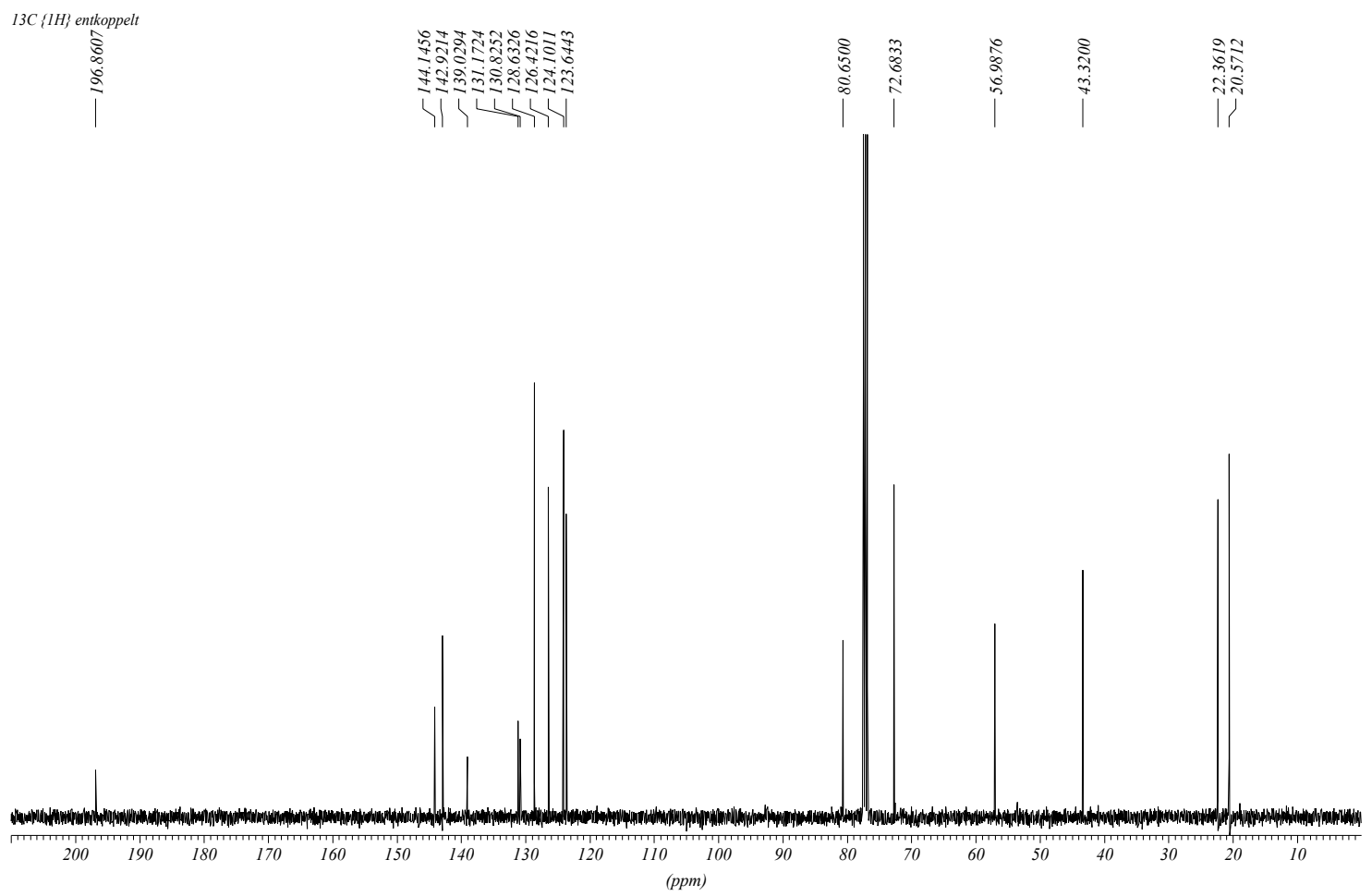

${ }^{13} \mathrm{C}$ NMR (100MHz, $\mathrm{CDCl}_{3}$ ): rac-Heliophenanthrone (3a) 